\title{
Ocorrência de sintomas de transtorno de ansiedade generalizada (TAG) e transtorno obsessivo-compulsivo (TOC) em estudantes de 11 a 18 anos de uma escola pública de Salvador
}

\author{
Occurrence of generalized anxiety disorder (GAD) and obsessive-compulsive disorder \\ $(O C D)$ in students aged 11 to 18 years from a public school in Salvador
}

\author{
Stefanio Emanoel Santos Tourinho ${ }^{1}$, Curt Hemanny ${ }^{2}$, Irismar Reis de Oliveira ${ }^{3 *}$ \\ ${ }^{1}$ Psicólogo. Mestrando do Programa de Pós-Graduação Processos Interativos dos Órgãos e Sistemas do ICS - UFBA.; \\ ${ }^{2}$ Psicólogo. Doutorado pelo Programa de Pós-Graduação Processos Interativos dos Órgãos e Sistemas do ICS - \\ UFBA. ; Professor Titular de psiquiatria. Programa de Pós-Graduação Processos Interativos dos Órgãos e Sistemas \\ do ICS - UFBA.
}

\begin{abstract}
Resumo
Introdução: o objetivo deste estudo é descrever a ocorrência de sintomas de TAG e TOC em adolescentes, com idade entre 11 e 18 anos, procedentes de escola pública da cidade do Salvador. Metodologia: estudo observacional e transversal, com 674 adolescentes de uma escola pública, que responderam a uma escala para avaliação de sintomas de TAG e TOC, aplicada entre o período de março e dezembro de 2015. Resultados: os escores da RCADS para TAG e TOC foram, em média, superiores aos pontos de corte estabelecidos pela escala, sugerindo aumento da presença desses sintomas na população estudada, com prevalência maior entre as alunas nos dois grupos de sintomas estudados. Conclusão: os dados apontam para a presença de sintomas de ansiedade generalizada e de TOC entre escolares, sugerindo uma maior necessidade de estudos para a compreensão do papel desses sintomas na qualidade de vida dessa população, como também sua importância como fatores de risco para o adoecimento mental. Medidas de prevenção devem direcionar os esforços para estudos metodologicamente mais robustos na área.

Palavras-chave: Ansiedade. Sintomas Obsessivo-Compulsivos. Saúde Escolar. Adolescente.
\end{abstract}

\begin{abstract}
Introduction: the aim of this study is to describe the occurrence of symptoms of GAD and OCD in adolescents, aged between 11 to 18 years old, from a public school in the city of Salvador. Methodology: observational and cross-sectional study with 674 adolescents from a public school, who responded to a scale for assessing symptoms of GAD and OCD applied from March to December 2015. Results: the RCADS scores for GAD and OCD were on average higher than the cutoff points established by the scale, suggesting an increased presence of these symptoms in the studied population, with a higher prevalence among girls in the two groups of symptoms studied. Conclusion: data point to the presence of symptoms of generalized anxiety and OCD among schoolchildren, suggesting a greater need for studies to understand the role of these symptoms in the quality of life of this population, as well as their importance as risk factors for mental illness. Prevention measures should direct efforts towards more methodologically robust studies in the area, Keywords: Anxiety. Obsessive-Compulsive Symptoms. School Health. Adolescent.
\end{abstract}

\section{INTRODUÇÃO}

No Brasil, o Estatuto da Criança e do Adolescente (ECA), Lei $\mathrm{N}^{\circ} 8.069$, de 1990, considera criança a pessoa com até 12 anos de idade incompletos e define a adolescência na faixa entre 12 a 18 anos. Para a Organização Mundial de Saúde (OMS), os limites cronológicos da adolescência estão entre 10 e 19 anos (ALLAIN-REGNAULT; BWIBO; CHIGIER, 1986).

A adolescência é uma fase de transição entre a saída da infância e o início da vida adulta, período que envolve diversas mudanças, tanto biológicas como emocionais,

Correspondente/Corresponding: *Irismar Reis de Oliveira - Instituto de Ciências da Sáude. Universidade Federal da Bahia - End: Av. Reitor Miguel Calmon, s/n, Vale do Canela, Salvador - BA. CEP: 40110-100. Tel: (71) 99981-9807 -E-mail: irismar@ufba.br psíquicas e ambientais (BLAKEMORE; MILLS, 2014). Apesar de ser um período de desenvolvimento da capacidade física e de adaptação às adversidades do meio (aumento da resiliência), a mortalidade, nesse período da vida, cresce $200 \%$, não sendo decorrente de doenças como câncer, infecções ou quadros cardiovasculares. 0 que ocorre nesse período e torna os adolescentes mais vulneráveis é a dificuldade de controle do comportamento e das emoções. Portanto, há um aumento considerável das taxas de acidentes, suicídio, depressão, ansiedade, homicídio e problemas relacionados ao uso de substâncias (DAHL, 2004). Acrescentamos que, nessa fase, o sujeito se depara com dúvidas e desafios diante das mudanças que experimentam, e os predispõem a alterações psicoafetivas (JOHNSON; CROSNOE; ELDER, 2011). 
Segundo Boarati et al. (2018), a adolescência implica uma transição psicossocial que engloba modificação de fatores biológicos decorrentes da puberdade. $\mathrm{O}$ indivíduo passa a ser mais autônomo, a apresentar aspectos mais definidos do que será a sua personalidade e a iniciar comportamentos interpessoais atrelados à sua sexualidade. É uma época importante para a aquisição de competências, mas também é fase de início dos principais quadros psiquiátricos, como o transtorno de ansiedade generalizada (TAG) e o transtorno obsessivo-compulsivo (TOC) (JONES, 2013). Antes da puberdade, os transtornos mentais são mais frequentes entre homens do que entre mulheres; após a puberdade, os transtornos passam a ser mais frequentes entre as mulheres. Estudos ingleses mostram que a prevalência no ano de transtornos mentais na adolescência pode chegar a 20\% (COWEN; HARRISON; BURNS, 2012).

O TAG é um transtorno mental caracterizado por preocupação contínua e excessiva (LEAHY, 2011). Segundo o DSM-5 (AMERICAN PSYCHIATRIC ASSOCIATION, 2014), essa preocupação excessiva envolve diversos eventos ou atividades e é desproporcional ao impacto real do evento antecipado. Portanto, o indivíduo tem dificuldade de controlar a preocupação excessiva e de evitar os pensamentos relacionados a essas situações, o que prejudica o seu funcionamento global.

Os sintomas do TAG são consistentes ao longo da vida. Entretanto, na adolescência as preocupações têm conteúdo mais voltado para temas relacionados à escola e ao desempenho esportivo. Na escola, especificamente, essas preocupações podem se expressar pela busca de perfeccionismo e comportamentos que demonstrem insegurança. Tais características podem resultar em perdas e no redirecionamento desses adolescentes, com intensificação do sofrimento psíquico (AMERICAN PSYCHIATRIC ASSOCIATION, 2014).

É importante saber a distinção entre transtornos que podem acompanhar ou se assemelhar ao TAG como, por exemplo, o TOC, que abordaremos a seguir. Indivíduos com TAG se apresentam ansiosos desde a infância, o que pode contribuir para o aparecimento de obsessões, entretanto, mais pesquisas são necessárias a respeito desse ponto. Os transtornos ansiosos tendem a anteceder o TOC, enquanto os transtornos afetivos tendem a sucedê-lo (PETRIBÚ, 2001).

Conforme o DSM-5 (AMERICAN PSYCHIATRIC ASSOCIATION, 2014), o TOC é caracterizado pela presença de obsessões e compulsões. As obsessões são pensamentos, impulsos ou imagens recorrentes e persistentes, causando a sensação de ansiedade, medo ou desconforto. As obsessões mais comuns envolvem medo de contaminação e dúvidas sobre situações que causam incerteza, imagens ou pensamentos de conteúdo violento ou sexual, que geralmente são vivenciados como intrusivos e egodistônicos, ou seja, seu conteúdo causa desprazer, medo e angústia (CORDIOLI, 2014).

Já as compulsões são caracterizadas por comportamentos repetitivos ou atos mentais que a pessoa se sente compelida a executar em resposta às obsessões. O objetivo é reduzir a ameaça, o desconforto e os riscos possivelmente associados às ideias obsessivas. Essas compulsões podem gerar rituais que ocupam grande parte do tempo, gerando déficit em seu comportamento habitual (CORDIOLI, 2014).

O TOC pode ter início na infância ou na adolescência. Quando não é tratado, seu curso é em geral crônico, com flutuação da sintomatologia ao longo da vida do sujeito. (AMERICAN PSYCHIATRIC ASSOCIATION, 2014). Por conta da cronicidade e de seu início precoce, torna-se importante aprofundar o entendimento sobre essa condição na adolescência, o que permitirá o desenvolvimento de práticas de intervenção nos estágios iniciais, visando ao diagnóstico e ao tratamento na fase inicial do adoecimento.

A prevalência do TAG na adolescência é muito variável. Um estudo brasileiro com amostra representativa da população de Taubaté, São Paulo, encontrou prevalência de $5,2 \%$ de transtorno de ansiedade em uma população de 7 a 14 anos, sendo duas vezes maior entre as meninas (POLANCZYK; LAMBERTE, 2014).

Um estudo foi realizado com 242 adolescentes na faixa etária entre 14 a 16 anos, 135 do sexo feminino e 106 do sexo masculino, em escolas públicas e privadas da cidade de Recife, com o intuito de identificar a prevalência de depressão e ansiedade em adolescentes. Constatou-se a prevalência de sintomas depressivos em 147 alunos ( $59,9 \%$ da amostra). Já a prevalência de adolescentes com ansiedade foi de $19,9 \%$, sinalizando para um risco aumentado de desenvolvimento de transtornos ansiosos e depressivos nessa população (JATOBÁ; BASTOS, 2007). A prevalência dos transtornos ansiosos em crianças e adolescentes está em torno de $4 \%$, sendo que o TAG varia entre $2,7 \%$ e 4,6\% (CASTILLO et al., 2000).

Os estudos epidemiológicos relatam prevalência, durante a vida, para transtornos de ansiedade em crianças e adolescentes variando entre 15 e 20\% (BEESDO; KNAPPE; PINE, 2009). Na adolescência, há uma heterogeneidade considerável dos sintomas mentais que podem evoluir para diversos transtornos psiquiátricos como transtornos de ansiedade (como o TAG), depressão e psicose. Os transtornos de ansiedade são mais prevalentes em meninas do que em meninos (WEHRY et al., 2015).

Em todo o mundo, há escassez de dados sobre a distribuição dos sintomas e transtornos mentais entre crianças e adolescentes. Esse problema no Brasil é ainda maior, principalmente na população de escolares. O conhecimento limitado nessa área, associado à importância da detecção precoce do sofrimento psíquico entre estudantes, justifica a realização de um estudo que busque observar a ocorrência desses sintomas entre os estudantes de ensino fundamental e médio. É importante salientar que nossas características socioeconômicas, envolvendo uma estrutura de desigualdade social profunda, nos levam à hipótese de uma prevalência elevada desses sintomas nessa população socialmente mais vulnerável. Mais estudos brasileiros no ambiente escolar e fora dele são 
importantes para melhores acolhimento, entendimento e orientação dessas crianças e adolescentes, bem como para avaliar a dimensão das sintomatologias.

O presente estudo objetiva descrever os sintomas de ansiedade generalizada e obsessivo-compulsivos em escolares de 11 a 18 anos, em uma escola da rede pública de Salvador, por meios da escala RCADS-47. Os sintomas serão estratificados pelas variáveis sexo e idade, permitindo a verificação da distribuição dos sintomas de TAG e TOC segundo essas variáveis sociodemográficas. Como foi mencionado anteriormente, a escolha da RCADS se deveu à sua característica de ser autoaplicável.

\section{METODOLOGIA}

Este estudo inclui estudantes de 11 a 18 anos de uma escola pública de Salvador, sendo parte da pesquisa Estudo da eficácia do Treinamento Cognitivo Processual em grupo (TCP-G) na prevenção de transtornos de ansiedade e depressão em adolescentes de escolas públicas municipais e privadas de Salvador: um ensaio clínico randomizado, aprovada pelo Comitê de Ética em Pesquisa da Maternidade Climério de Oliveira (Universidade Federal da Bahia) e tendo como instituição proponente o Sanatório São Paulo, com número de processo CAAE: 42264315.0.0000.5543.

Obteve-se o consentimento da Secretaria de Educação do Município de Salvador e a direção da escola municipal foi informada sobre a natureza do estudo, permitindo, a realização da coleta dos dados. Os pais dos adolescentes foram informados sobre as características do estudo, seus benefícios, riscos e possíveis danos, tendo assinado o Termo de Consentimento Livre e Esclarecido (TCLE). O mesmo procedimento foi realizado com os alunos, que assinaram o termo de assentimento. $O$ presente estudo utiliza os dados coletados no primeiro encontro com os estudantes. Cada participante recebeu um caderno com questionários a serem preenchidos. Os pesquisadores participantes foram treinados para auxiliar os estudantes no preenchimento desses instrumentos, que durou aproximadamente 40 minutos.

A RCADS é uma adaptação norte-americana da Spence's Children Anxiety Scale (SCAS), de origem Australiana. A SCAS avalia sete dimensões: pânico e agorafobia, fobia social, ansiedade de separação, ansiedade generalizada, obsessão e compulsão, medo de agressão física e desejabilidade social.

A escala RCADS é constituída de 47 itens, desenvolvidos para medir sintomas relevantes dos transtornos de ansiedade (Transtorno de Ansiedade Generalizada, Transtorno de Ansiedade de Separação, Transtorno de Pânico, Transtorno Obsessivo-Compulsivo e Fobia Social) e do transtorno depressivo maior (TDM) em crianças, de acordo com o DSM-IV (EBESUTANI et al., 2012). É pontuado numa escala tipo likert de 4 pontos $(0=$ nunca, $1=$ às vezes, $2=$ frequente e $3=$ sempre). É composta por 37 itens de ansiedade e 10 de depressão. Os pontos de corte para as subescalas foram estabelecidos pelos autores da seguinte forma: TAS, 5 pontos; FS, 10 pontos; pânico, 12 pontos; TAG, 7 pontos; TOC, 5 pontos e TDM, 11 pontos (SANDÍN; VALIENTE; CHOROT, 2009).

A RCADS-47 foi submetida à validação semântica por estudo realizado em Salvador e Curitiba (NOVA, 2016). Em 2002, a RCADS-47 foi submetida a uma amostra populacional de 405 crianças e adolescentes, oriundas de escolas não governamentais da Austrália. $\mathrm{O}$ objetivo do estudo foi examinar as propriedades psicométricas da RCADS. A consistência interna se mostrou adequada, assim como a validade de convergência que foi boa, demonstrada através de correlações moderadas a fortes, com outras escalas de avaliação de estados afetivos, como a Revised Children's Manifest Anxiety Scale, RCMAS, e a Children's Depression Inventory, CDI (DE ROSS; GULLONE; CHORPI$T A, 2002)$. A consistência interna da RCADS também foi confirmada em avaliação no Havaí, em uma população de 513 crianças e adolescentes, bem como a adequação ao modelo de seis fatores (CHORPITA; MOFFITT; GRAY, 2005).

Realizou-se uma análise descritiva dos dados coletados, com estratificação para sexo e idade. Foi utilizado o programa estatístico R (COUTO; REIS; OLIVEIRA, 2016; SILVA DA; DINIZ; BORTOLUZZI, 2009).

\section{RESULTADOS}

Na Tabela 1, encontramos os valores referentes à distribuição da amostra para sexo e a pontuação da escala RCDAS-47 total para os dois grupos de sintomas, ainda não estratificada por sexo.

Tabela 1 - Características clínicas da amostra

\begin{tabular}{lc}
\hline $\begin{array}{l}\text { Idade M (DP) } \\
\text { Sexo }\end{array}$ & $14,31 \quad(1,81)$ \\
$\quad$ Feminino $\mathrm{n}(\%)$ & $351 \quad(52,2)$ \\
$\quad$ Masculino $\mathrm{n}(\%)$ & $322(47,8)$ \\
RCADS-47 & \\
$\quad$ Sintomas de TAG M (DP) & $8,46(4,072$ \\
$\quad$ Sintomas de TOC M (DP) & $6,91(3,955)$ \\
\hline
\end{tabular}

Nota: $M=$ Média, $D P=$ Desvio Padrão; $T A G$ = Transtorno de Ansiedade Generalizada; $T O C=$ Transtorno Obsessivo-Compulsivo

Fonte: Dados da pesquisa

Na Tabela 2, observa-se a média e o desvio padrão dos escores da RCADS para os sintomas de TAG e TOC, diferenciados por sexo. Os escores para ambos os grupos de sintomas foram maiores para o sexo feminino. Foi utilizado como marcador da presença de sintomas o escore de corte da RCADS para TAG de 7, e para a presença dos sintomas de TOC de 5. Encontramos um escore médio para as mulheres em relação aos sintomas de TAG de 9,34, significando um aumento dos sintomas ansiosos na população de escolares, principalmente entre as mulheres. 
Tabela 2 - Valores que indicam o tamanho amostral, a média e o desvio padrão de cada diagnóstico de acordo com o sexo.

\begin{tabular}{lcc}
\hline \multicolumn{3}{c}{ RCADS TAG } \\
\hline Sexo & $\mathbf{N}$ & $\mathbf{M}(\mathbf{D P})$ \\
Feminino & 341 & $9,34(3,992)$ \\
Masculino & 302 & $7,47(3,944)$ \\
\hline \multicolumn{3}{c}{ RCADS TOC } \\
\hline Sexo & $\mathbf{N}$ & $\mathbf{M}(\mathbf{D P})$ \\
Feminino & 341 & $7,61(4,019)$ \\
Masculino & 303 & $6,13(3,736)$ \\
\hline
\end{tabular}

Nota: $N=$ Frequência; $M=$ Média, $D P=$ Desvio Padrão; $R C A D S=$ Revised Children's Anxicty and Depression; TAG = Transtorno de Ansiedade Generalizada; TOC = Transtorno Obsessivo-Compulsivo

Fonte: Dados da pesquisa

Tabela 3 - Correlações bivariadas entre sintomas, idade e sexo

\begin{tabular}{lcc}
\hline \multicolumn{1}{c}{ Variáveis } & Sintomas de TAG & Sintomas de TOC \\
\hline Idade & $0,111^{*}$ & 0,017 \\
Sexo (Feminino) & $0,230^{* *}$ & $0,187^{* *}$ \\
Sintomas de TAG & - & $0,625^{* *}$ \\
$* p \leq 0,05$ & & \\
$* * p \leq 0,0005$ & & \\
\hline
\end{tabular}

Nota: $T A G=$ Transtorno de Ansiedade Generalizada $;$ TOC $=$ Transtorno Obsessivo-Compulsivo

Fonte: Dados da pesquisa

A Tabela 3 apresenta as correlações bivariadas entre os escores dos sintomas entre si e as correlações com idade e sexo. Os sintomas de ansiedade generalizada e transtorno obsessivo- compulsivo apresentaram correlação positiva forte ( $r$ de Pearson $=0,626 ; p<0,0005$ ), isto é, os sintomas de TAG tendem a aumentar juntamente com os sintomas de TOC.

A idade também foi correlacionada positivamente com a presença de sintomas de TAG ( $r$ de Pearson $=0,111$; $p=0,0005)$. O sexo feminino está mais correlacionado com a presença de sintomas de TAG ( $r$ de Pearson = 0,230; $p<$ $0,0005)$ e TOC ( $r$ de Pearson $=0,187 ; p<0,0005$ ).

\section{DISCUSSÃO DOS RESULTADOS}

O presente estudo, que analisou 674 estudantes de uma escola pública em Salvador, mostrou uma pontuação média da RCADS para TAG de 9,34 para o sexo feminino e 7,47 para o sexo masculino. Considerando o ponto de corte da escala para TAG de 7, encontramos um aumento dos sintomas de ansiedade generalizada para essa população. Sendo que a correlação mostrou mais sintomas entre as mulheres, quando comparadas aos alunos. Em relação aos sintomas de TOC entre os estudantes avaliados, encontramos uma média de 7,61 para mulheres e de 6,13 para homens, representando um aumento de sintomas do TOC nesses estudantes, tomando como parâmetro o ponto de corte da RCADS de 5. Portanto, em ambos os transtornos analisados, o estudo mostrou uma maior ocorrência de sintomas na população estudada.

Uma pesquisa realizada em 2010, nos Estados Unidos, pelo National Comorbidity Survey-Adolescent Supplement (NCS-A) encontrou resultados semelhantes aos nossos em relação ao TAG, com maior prevalência entre as mulheres. Foi uma pesquisa face a face, com representatividade nacional, que avaliou 10.123 adolescentes com idades entre 13 e 18 anos. Os transtornos de ansiedade foram a condição mais comum (31,9\%), sendo que quase um em cada três adolescentes preencheram os critérios. As taxas de transtornos individuais variaram de 2,2\% para TAG a $19,35 \%$ para fobia específica. Todos os subtipos de transtorno de ansiedade foram mais frequentes em mulheres, seguidos por transtornos de comportamento $(19,1 \%)$, transtornos do humor $(14,3 \%)$ e transtornos por uso de substâncias $(11,4 \%)$. A presença de comorbidade nessa amostra foi de $40 \%$, ou seja, a chance de um estudante com transtorno de ansiedade apresentar critérios para outra patologia mental foi grande nesse estudo. A prevalência geral de transtornos com comprometimento e (ou) sofrimento grave foi de $22,2 \%$ (11,2\% com transtornos de humor; $8,3 \%$ com transtornos de ansiedade; $9,6 \%$ com transtornos de comportamento). A idade média de início dos transtornos foi mais precoce para ansiedade (6 anos), seguida de 13 anos para o início dos transtornos de humor e 15 anos para os transtornos por uso de substâncias (MERIKANGAS et al., 2010). Além de fornecer estimativas de prevalência, os resultados também confirmam observações de investigações anteriores em adolescentes, nos EUA e em outros países, sobre a associação de características sociodemográficas e transtornos mentais. Em particular, as adolescentes foram mais propensas do que os meninos a ter transtornos de humor e de ansiedade.

Os dados epidemiológicos sobre o TOC, nas últimas décadas, mostram uma prevalência de 1 a $4 \%$ desse transtorno entre crianças, adolescentes e adultos, com um impacto importante no funcionamento global dessas pessoas. Na grande maioria dos casos, os sintomas se iniciam antes dos 18 anos, podendo ter início infância (NAZEER et al., 2020). Os estudos recentes sobre a carga global das doenças em crianças e adolescentes apontam os transtornos mentais como a maior causa de anos vividos com incapacidade. A OMS reconhece a grande necessidade de dados específicos por sexo e idade em relação à saúde mental das crianças. Poucos países no mundo possuem esses dados de forma sistemática. Um estudo dinamarquês realizou o seguimento de 1,3 milhão de crianças nascidas entre 1995 e o final de 2016. Observou-se que o transtorno de maior incidência antes dos 18 anos de idade, em mulheres, foi o transtorno de ansiedade. Vários transtornos apresentaram maior risco de ocorrência na infância e na adolescência entre as mulheres, inclusive o TOC. Como esse estudo acompanhou essas crianças e adolescentes da infância até os dezoitos anos, os autores 
puderam observar que o risco de transtorno de ansiedade, entre os 6 e 13 anos, foi maior entre homens, sendo que esse padrão se reverte antes dos dezoitos anos, passando a ser mais frequente entre as mulheres. A incidência do TOC nesse estudo, teve um pico em ambos os sexos no início da adolescência, o que foi considerado pelos autores como um achado não consistente com a literatura, que mostra esse pico ocorrendo mais tardiamente na adolescência (DALSGAARD et al., 2020).

Essas observações nos levam à primeira limitação importante de nosso estudo. Em nossa análise, não foi possível estratificar os escores da RCADS-47 considerando a fase inicial da adolescência e a adolescência tardia. Como vimos nos dados acima, a distribuição da incidência e, possivelmente, da prevalência dos transtornos mentais durante esses anos da infância e adolescência pode variar de acordo com a fase do desenvolvimento das crianças e dos adolescentes. Uma segunda limitação foi o fato de este estudo ser de corte transversal, impossibilitando a avaliação da incidência desses transtornos na nossa população, como também não permitindo a análise de variáveis confundidoras, que pudessem modificar os resultados encontrados. Há necessidade de dados mais consistentes sobre a epidemiologia dos transtornos mentais entre as crianças e adolescentes, considerada de importância central pela OMS, como observamos anteriormente. Para tanto, são necessários estudos de seguimento dessa população entre nós. Mesmo assim, apesar dessas limitações, as observações do presente estudo apontam para a necessidade de políticas públicas direcionadas aos transtornos mentais em escolares, principalmente pelos achados na literatura, que colocam essa fase da vida como o período de início da maioria das patologias mentais.

\section{CONCLUSÃO}

O presente estudo traz importantes informações sobre a saúde mental de escolares entre 11 e 18 anos, período de início da maioria das patologias mentais. Nossos dados sobre a ocorrência de sintomas de ansiedade e de sintomas obsessivo-compulsivos nessa população são de importância central, considerando que eles podem indiciar modificações iniciais nos processos de maturação cerebral, correspondendo a fatores de risco para o adoecimento psíquico em fases posteriores da vida. Portanto, o conhecimento sobre a prevalência desses sintomas entre crianças e adolescentes permitirá a construção de estratégias de prevenção primária e secundária na saúde mental. As limitações dos nossos achados, entretanto, apontam para a necessidade urgente de estudos metodologicamente mais robustos e estratégias de saúde pública que, de imediato, priorizem o cuidado dirigido a esses grupos específicos.

\section{REFERÊNCIAS}

ALLAIN-REGNAULT, M.; BWIBO, N. O.; CHIGIER, E. Young people's health - A challenge for society. World Health Organization;Technical Report Series, Geneva, v. n. 731, p. 1-117, 1986.

AMERICAN PSYCHIATRIC ASSOCIATION. Manual diagnóstico e estatístico de transtornos mentais: DSM-5. Porto Alegre: Artmed, 2014.

BEESDO, K.; KNAPPE, S.; PINE, D. S. Anxiety and anxiety disorders in children and adolescents: Developmental issues and implications for DSM-V. Psychiatric Clinics of North America, Philadelphia, v. 32, n. 3, p. 483-524, 2009.

BLAKEMORE, S. J.; MILLS, K. L. Is adolescence a sensitive period for sociocultural processing? Annual Review of Psychology, Palo Alto, v. 65, n. August 2013, p. 187-207, 2014.

BOARATI, M. A.; KAUSE, R. N.; FELÍCIO, J. L. Psiquiatria na adolescência. In: MELEIRO, A. (Ed.). Psiquiatria: estudos fundamentais. Rio de Janeiro: Guanabara Koogan, 2018. p. 545-558.

BRASIL. Lei $n^{\circ} 8.069$, de 13 de julho de 1990. Dispõe sobre o Estatuto da Criança e do Adolescente e dá outras providências. Diário Oficial [da] União. Brasília, DF, 1990. Disponível em: http://www.planalto.gov.br/ ccivil_03/leis/l8069.htm.

CASTILLO, A. R. G. et al. Transtorno de ansiedade. Rev. Bras. Psiquiatr., São Paulo, v. 22, p. 20-23, 2000.

CHORPITA, B. F.; MOFFITT, C. E.; GRAY, J. Psychometric properties of the Revised Child Anxiety and Depression Scale in a clinical sample. Behav. Res. Ther., Oxford, v. 43, n. 3, p. 309-322, 2005

CORDIOLI, A. V. Manual de terapia cognitivo-comportamental para o transtorno obsessivo-compulsivo. Porto Alegre: Artmed, 2014.

COUTO, I. S. L.; REIS, D. M. L.; OLIVEIRA, I. R. DE. Prevalência de sintomas de depressão em estudantes de 11 a 17 anos da rede pública de ensino de Salvador. Rev. de Ciênc. Méd.e Biol., Salvador, v. 15, n. 3 , p. 370-374, 2016.

COWEN, P. et al. (Eds.) Shorter Oxford Textbook of Psychiatry. 6. ed. Oxford: Oxford University Press, 2012

CUMMINGS, C. M.; CAPORINO, N. E.; KENDALL, P. C. Adolescents: 20 years after. Psychol. Bull., Washington, v. 140, n. 3, p. 816-845, 2014.

DAHL, R. E. Adolescent brain development: A period of vulnerabilities and opportunities. Ann. N.Y. Acad. Sci., New York, v. 1021, p. 1-22, 2004.

DALSGAARD, S. et al. Incidence rates and cumulative incidences of the full spectrum of diagnosed mental disorders in childhood and adolescence. Jama Psychiatry, Chicago, v. 77, n. 2, p. 155-164, 2020.

DE ROSS, R. L.; GULLONE, E.; CHORPITA, B. F. The revised child anxiety and depression scale: A psychometric investigation with Australian youth. Behaviour Change, Australia, v. 19, n. 2, p. 90-101, 2002.

EBESUTANI, C. et al. The revised child anxiety and depression scaleshort version: Scale reduction via exploratory bifactor modeling of the broad anxiety factor. Psychological Assessment, Arlington, v. 24, n. 4, p. 833-845, 2012.

JATOBÁ, J. D. V. N.; BASTOS, O. Depressão e ansiedade em adolescentes de escolas públicas e privadas. J. Adolesc. Res., Tucson, v. 56, n. 3, p. 171-179, 2007

JOHNSON, M. K.; CROSNOE, R.; ELDER, G. H. Insights on adolescence from a life course perspective. J. Adolesc. Res., Tucson, v. 21, n. 1, p. 273-280, 2011 
JONES, P. B. Adult mental health disorders and their age at onset. Br. J. Psychiatr., London, v. 202, n. Suppl. 54, 2013.

LEAHY, R. L. Livre de ansiedade. Porto Alegre: Artmed, 2011.

MERIKANGAS, K. R. et al. Lifetime prevalence of mental disorders in US adolescents: Results from the national comorbidity study-adolescent supplement (NCS-A). J. Am. Acad. Child Adolesc. Psychiatry, EUA, v. 49, n. 10, p. 980-989, 2010.

NAZEER, A. et al. Obsessive-compulsive disorder in children and adolescents: epidemiology, diagnosis and management. Translational Pediatrics, Hong Kong, v. 9, n. S1, p. S76-S93, 2020.

NOVA, I. S. V. Análise psicométrica da Revised Child Anxiety And Depression Scale Reduzida (RCADS-25). Salvador: Universidade Federal da Bahia, 2016.

PETRIBÚ, K. Comorbidade no transtorno obsessivo-compulsivo. Revista Brasileira de Psiquiatria, São Paulo, v. 23, suppl 2, p. 17-20, 2001.
POLANCZYK, G. V.; LAMBERTE, M. T. M. R. Psiquiatria da infância e adolescência. São Paulo: Manole, 2014.

SANDÍN, B.; VALIENTE, R. M.; CHOROT, P. RCADS: evaluación de los síntomas de los trastornos de ansiedad y depresión en niños y adolescentes. Revista de Psicopatología y Psicología Clínica, España, v. 14, n. 3, 2009.

SILVA (da), B. F.; DINIZ, J.; BORTOLUZZI, M. A. Minicurso de estatística básica: introdução ao software R. Samta Maria, RS: Universidade de Santa Maria, 2009. 100 p.

WEHRY, A. M. et al. Assessment and treatment of anxiety disorders in children and adolescents. Curr Psychiatry Rep., Philapdelphia, v. 17, n. 7, p. 1-19, 2015.

Submetido em: 07/12/2020

Aceito em: 14/12/2020 